\title{
Quantum anisotropic Heisenberg chains with superlattice structure: a DMRG study.
}

\author{
J. Silva-Valencia, ${ }^{1,2}$ J. C. Xavier, ${ }^{2}$ and E. Miranda ${ }^{2}$ \\ ${ }^{1}$ Departamento de Fúsica, Universidad Nacional de Colombia, A. A. 5997, Bogotá, Colombia \\ ${ }^{2}$ Instituto de Física Gleb Wataghin, Unicamp, Caixa Postal 6165, 13083-970 Campinas SP, Brazil
}

(Dated: 28th September 2018)

\begin{abstract}
Using the density matrix renormalization group technique, we study spin superlattices composed of a repeated pattern of two spin- $1 / 2$ XXZ chains with different anisotropy parameters. The magnetization curve can exhibit two plateaus, a non trivial plateau with the magnetization value given by the relative sizes of the sub-chains and another trivial plateau with zero magnetization. We find good agreement of the value and the width of the plateaus with the analytical results obtained previously. In the gapless regions away from the plateaus, we compare the finite-size spin gap with the predictions based on bosonization and find reasonable agreement. These results confirm the validity of the Tomonaga-Luttinger liquid superlattice description of these systems.
\end{abstract}

PACS numbers: 71.10.Pm, 75.45.+j, 75.60.Ej

\section{INTRODUCTION}

The synthesis of new materials which can be described in terms of spin chains or spin ladders has revived the study of quantum spin systems in one dimension in the last few years $\frac{1}{n}$ These systems are known to have surprising features such as the presence of a gap in isotropic Heisenberg chains of integer spins and its absence when the spins are half-integers ${ }^{2,3,4,5.6 .7}$ More recently, many experimental and theoretical results have shown the presence of plateaus, in which the magnetization in an external magnetic field is quantized to fractions of the saturated value ${ }^{8.9,10,11,12,13}$ These systems have special spatial structures, such as p-merization or ladder geometry, which are responsible for the appearance of the magnetization plateaus. Oshikawa, Yamanaka and Affleck ${ }^{14}$ derived the condition $p\left(S-m^{z}\right)=$ integer, necessary for the appearance of the magnetization plateaus in $1 \mathrm{D}$ systems. Here, $p$ is the number of sites in the unit cell of the magnetic ground state, $S$ is the magnitude of the spin and $m^{z}$ is the magnetization per site (taken to be in the $z$-direction). The plateau state can be viewed as a gapped state with nonzero magnetization, the integer spin chains being a special case where $p=1$ and $m^{z}=0$.

Other types of spatial structures, such as quasiperiodic couplings,,$\frac{15}{5}$ an inhomogeneous magnetic field ${ }^{16}$ or a superlattice structure $\frac{17}{17}$ can also give rise to magnetization plateaus. The superlattice case with periodic boundary conditions was studied by two of us in a previous work 17 There, we considered a spin superlattice (SS) composed of a repeated pattern of two long and different spin- $\frac{1}{2} \mathrm{XXZ}$ chains. This model can be viewed as the limit of $p$-merized chains when the number of sites per unit cell is very large. Magnetization plateaus were found, with magnetization values that depend on the relative sizes of the sub-chains, in accordance with the condition of Ref. 14. The determination of the width and magnetization values of the plateaus relied on the Bethe Ansatz exact solution of the XXZ chain ${ }^{18}$ The low-energy properties in the gapless regions away from the plateaus, however, could be described by bosoniza- tion in terms of a Tomonaga-Luttinger liquid superlattice (TLLS) $\stackrel{19}{\underline{1}}$ This type of system is of potential interest in nanoelectronic applications, where nanowire superlattice structures have been built with semiconducting carbon nanotubes ${ }^{20}$ A TLLS would be obtained if the metallic analogue could be manufactured.

In this work, it is our purpose to check numerically the analytical predictions based on the Bethe Ansatz and bosonization using the density matrix renormalization group (DMRG) ${ }^{6.21}$ In particular, we calculate the magnetization curve, characterize its plateaus and determine the effective Tomonaga-Luttinger liquid parameters for spin superlattices. The unit cell of each SS consists of two $S=1 / 2 \mathrm{XXZ}$ sub-chains with different anisotropy parameters $\Delta_{\lambda}$ and sizes $L_{\lambda}(\lambda=1,2)$ (but the same planar coupling), as shown schematically in Fig. 10 The Hamiltonian of the SS is written as a sum over $N_{c}$ unit cell Hamiltonians

$$
H_{S S}=\sum_{j=0}^{N_{c}-1} H_{c}\left(j, \Delta_{1}, L_{1}, \Delta_{2}, L_{2}\right) .
$$

Each unit cell, on the other hand, consists of a total of $L_{1}+L_{2}-2=L_{c}$ bonds. The first $L_{1}-1$ bonds have anisotropy parameter $\Delta_{1}$ and the following $L_{2}-1$ bonds have anisotropy parameter $\Delta_{2}$, with Hamiltonian

$$
\begin{aligned}
H_{c}\left(j, \Delta_{1}, L_{1}, \Delta_{2}, L_{2}\right) & =\sum_{n=1}^{L_{1}-1} H\left(j L_{c}+n, \Delta_{1}\right) \\
& +\sum_{n=1}^{L_{2}-1} H\left(j L_{c}+L_{1}+n-1, \Delta_{2}\right),
\end{aligned}
$$

where

$$
H(n, \Delta)=S_{n}^{x} S_{n+1}^{x}+S_{n}^{y} S_{n+1}^{y}+\Delta S_{n}^{z} S_{n+1}^{z} .
$$

In the last expression, $S_{n}^{x}, S_{n}^{y}$ and $S_{n}^{z}$ are spin- $\frac{1}{2}$ operators at the $n$-th site. The total number of sites in the SS is, for open boundary conditions, $L=\left(L_{1}+L_{2}-2\right) N_{c}+1$ and, for periodic boundary conditions, $L=\left(L_{1}+L_{2}-2\right) N_{c}$. 


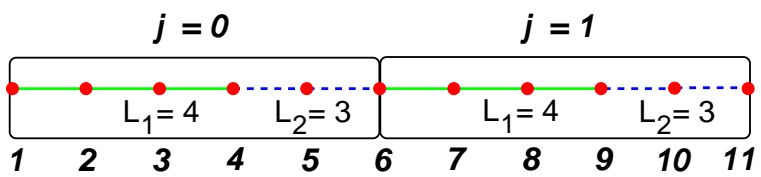

Figure 1: Spin superlattice structure with two unit cells $\left(N_{c}=2\right), L_{1}=4$, and $L_{2}=3$. The solid bonds correspond to $\Delta_{1}$ and the dashed ones to $\Delta_{2}$. The total number of sites is $L=\left(L_{1}+L_{2}-2\right) N_{c}+1=11$ (open boundary conditions).

We have set the transverse coupling to 1 to set the energy scale. Since we will be interested in increasing both $L_{1}$ and $L_{2}$ while keeping their ratio fixed, we define $P$ as the greatest common divisor of $L_{1}$ and $L_{2}$ and study the behavior of the system as $P$ grows. Note that each subchain contains $L_{\lambda}-1$ bonds and $L_{\lambda}$ spins. The spins at the boundaries of the sub-chains, however, should be viewed as belonging to either sub-chain (see Fig. 1). Of course, in the limit of large $L_{\lambda}$ in which we are interested, this ambiguity at the boundaries is immaterial. Finally, the total Hamiltonian has an external magnetic field $h$ applied along the anisotropy $z$-axis

$$
H_{T}=H_{S S}-\sum_{n=1}^{L} h S_{n}^{z} .
$$

Let us first recall some known results on the XXZ model, which is the basic building block of the SS. Using the Bethe Ansatz, Yang and Yang 18 found the exact solution of the one-dimensional $S=1 / 2$ anisotropic (XXZ) Heisenberg model. They showed that the model may exhibit three phases, according to the value of the Ising anisotropy $\Delta$ : a ferromagnetic (FM) phase for $\Delta<-1$, a Néel antiferromagnetic (AFM) phase with a spin gap for $\Delta>1$, and a gapless (critical) phase for $-1<\Delta<1$. The low-energy properties of the gapless phase can be described in terms of a Tomonaga-Luttinger liquid ${ }^{22}$ with velocity $u$ and interaction parameter $K^{23}$

\section{MAGNETIZATION PLATEAUS}

In the Hamiltonian (2), the magnetic field couples to a conserved quantity $S_{t o t}^{z}=\sum_{n} S_{n}^{z}$. Thus, to obtain the magnetization curve we only need the ground state energy at $h=0, E\left(S_{t o t}^{z}, h=0\right)$, in each of the subspaces with fixed total spin projection $S_{\text {tot }}^{z} \in\{0,1, \ldots, L / 2\}$. Then, we can readily obtain the energy in a finite magnetic field $h$ through the relation $E\left(S_{t o t}^{z}, h\right)=E\left(S_{t o t}^{z}, 0\right)-$ $h S_{t o t}^{z}$, from which we can construct the magnetization curve. 24

Since the DMRG is more precise and computationally faster with open boundary conditions the magnetization curve was calculated this way. We considered lattice sizes up to 160 sites keeping up to $m=150$ states per block. The discarded weight was kept around $10^{-12}$. On

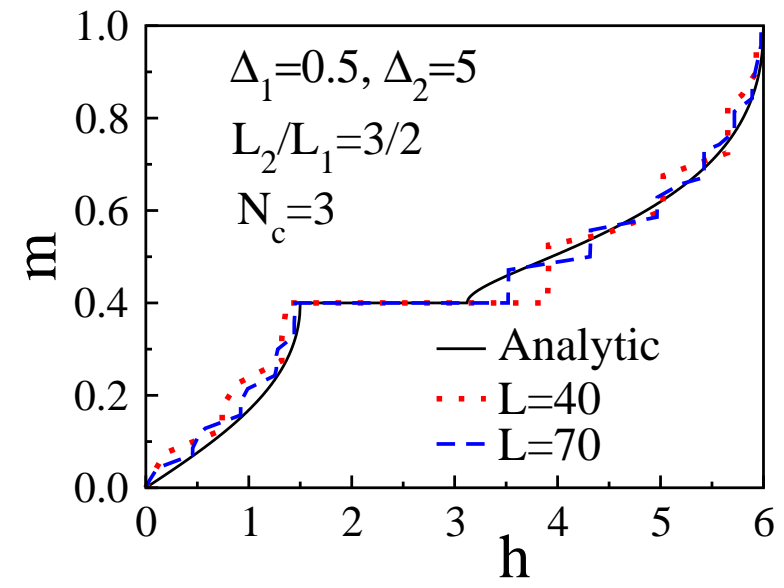

Figure 2: Magnetization density of a spin superlattice with $\Delta_{1}=0.5, \Delta_{2}=5, L_{1}=2 P, L_{2}=3 P(\ell=3 / 2)$, and $N_{c}=3$. The short-dashed line is for $P=3(L=40)$, and the long-dashed one for $P=5(L=70)$. The solid line was obtained analytically for a spin superlattice with long unit cells $(P \gg 1), 17$

the other hand, in order to compare with the analytical predictions of the Tomonaga-Luttinger liquid parameters which were obtained with periodic boundary conditions, we have also analyzed SS's with the latter boundary conditions. In order to obtain a comparable accuracy, we considered chains with up to 100 sites with up to $m=600$ states per block. The truncation errors were below $10^{-9}$.

Fig. 2] shows the magnetization density $m$ (magnetization per site normalized to the saturation value) of a SS as a function of the external magnetic field for $\Delta_{1}=0.5$ and $\Delta_{2}=5$. We set $N_{c}=3$, since we have observed that the magnetization curve is fairly insensitive to the number of unit cells. The sub-lattice sizes were chosen such that $L_{2} / L_{1} \equiv \ell=3 / 2$. Two lattice sizes are shown in the figure: $L=40$ and $L=70$. They correspond to $L_{1}=2 P$ and $L_{2}=3 P$, with $P=3$ and $P=5$, respectively. For both sizes the magnetization density has a plateau at $m=0.4$.

The continuous line in Fig. 2 corresponds to the case where we consider two long sub-lattices with a fixed size ratio $(P \gg 1)$. In this case, the magnetization density of the $\mathrm{SS}$ is given by $\underline{17}$

$$
m=\frac{L_{1} m_{1}+L_{2} m_{2}}{L_{1}+L_{2}}
$$

where $m_{\lambda}$ is the magnetization per site of sub-chain $\lambda$. The magnetization density of each sub-lattice was obtained from the Bethe Ansatz solution in Ref. 17.

It can be seen that the three curves have a magnetization plateau at $m=0.4$. This has been shown to correspond to $m_{1}=1$ (FM phase) and $m_{2}=0$ (AFM phase) in Eq. (3) $\frac{17}{1}$ The plateau is a result of the "spin incompressibility" of both sub-chains: sub-chain 1 is magnetized at saturation, whereas sub-chain 2 has a spin gap. However, 


\begin{tabular}{cccc}
\hline \hline$L$ & $h_{s}$ & $h_{c}$ & $\Gamma_{N T}$ \\
\hline 10 & 0.67719 & 5.17056 & 4.49336 \\
40 & 1.35468 & 3.90474 & 2.55006 \\
70 & 1.44854 & 3.51944 & 2.07089 \\
100 & 1.47166 & 3.36001 & 1.88835 \\
130 & 1.48329 & 3.27965 & 1.79635 \\
160 & 1.48900 & 3.23373 & 1.74473 \\
extrapolated & 1.495 & 3.146 & 1.667 \\
prediction & 1.5 & 3.121 & 1.621 \\
\hline \hline
\end{tabular}

Table I: The saturation field $h_{s}$, the critical field $h_{c}$ and the width of plateau $\Gamma_{N T}$ at $m=0.4$ as a function of lattice size. For all sizes $\Delta_{1}=0.5, \Delta_{2}=5, L_{1}=2 P, L_{2}=3 P(\ell=3 / 2)$, and $N_{c}=3$. The second to last line is the VBS extrapolation and the last one is the prediction of Ref. 17 .

the magnetic field width of the plateau is dependent on the sub-chain sizes $L_{1}$ and $L_{2}$. In fact, the fields at each end of the plateau are approached asymmetrically as the system grows. The critical field $h_{c}$ (right-hand side of the plateau) shows a larger finite size error than the saturation field $h_{s}$ (left-hand side of the plateau).

The behavior of the saturation field $h_{s}$, critical field $h_{c}$ and the width of the plateau $\Gamma_{N T}=h_{c}-h_{s}$ as a function of the lattice size $L$ is shown in Table \ Again we focus on the plateau at $m=0.4$ and $\ell=3 / 2\left(L_{1}=2 P\right.$, $L_{2}=3 P$, and $\left.N_{c}=3\right), \Delta_{1}=0.5$, and $\Delta_{2}=5$. We have found the thermodynamic limit of these quantities through numerical extrapolation using the Vanden Broeck-Schwartz (VBS) algorithm, ${ }^{25}$ which is shown in the bottom line. The saturation field increases with the lattice size, but slowly at large $L$, as seen in the first column of Table I Extrapolation to infinite $L$ yields the value $h_{s}=1.495$. As we have seen in connection with Eq. (3), we have $m_{1}=1$, i.e., the sub-lattice 1 is totally magnetized. As shown in Ref. 17, the saturation field is the field at which the corresponding homogeneous chain with the same anisotropy parameter reaches saturation. For $\Delta_{1}=0.5$, this happens at $h=1+\Delta_{1}=1.5$, very close to the numerical value of $h_{s}$ and compatible with our interpretation.

In the second column of Table【 it can be seen that the critical field $h_{c}$ decreases with the lattice size. The convergence is slower than for $h_{s}$ due to the smaller derivative of the magnetization as a function of the field as the plateau is approached from above, as is apparent in the analytical result of Fig. 2] The extrapolated infinite size value of the critical field is $h_{c}=3.146$. This should be compared with the gap of a homogeneous lattice with anisotropy parameter $\Delta_{2}=5$, which is $h=3.121$. This value is close to the numerically determined critical field. This agreement is again consistent with the sub-lattice 2 with anisotropy parameter $\Delta_{2}=5$ being in an AFM spin-gapped phase. The width of plateau $\Gamma_{N T}=h_{c}-h_{s}$ as a function of $L$ is shown in the third column of Table The extrapolated infinite size limit of the plateau width is $\Gamma_{N T}^{\infty}=1.667$.

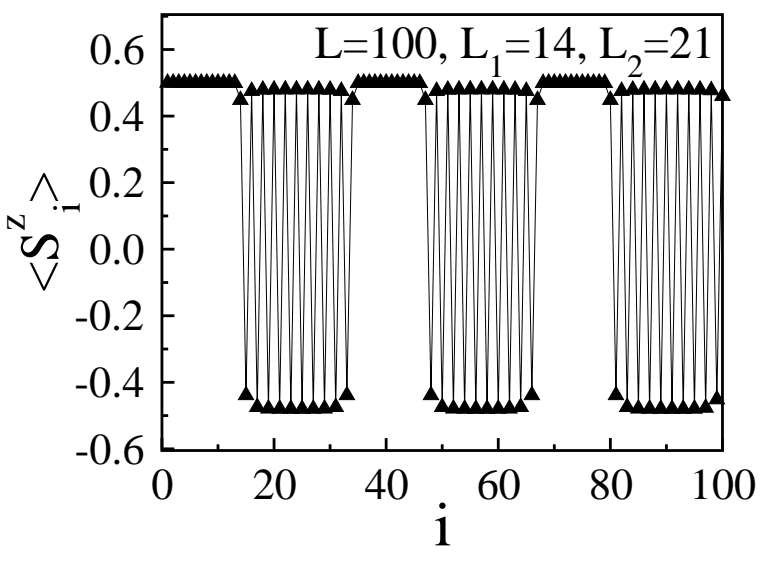

Figure 3: The magnetization profile of a spin superlattice at the magnetization plateau of $m=0.4$. The parameters are $\Delta_{1}=0.5, \Delta_{2}=5, L_{1}=14$, and $L_{2}=21(\ell=3 / 2, L=100)$.

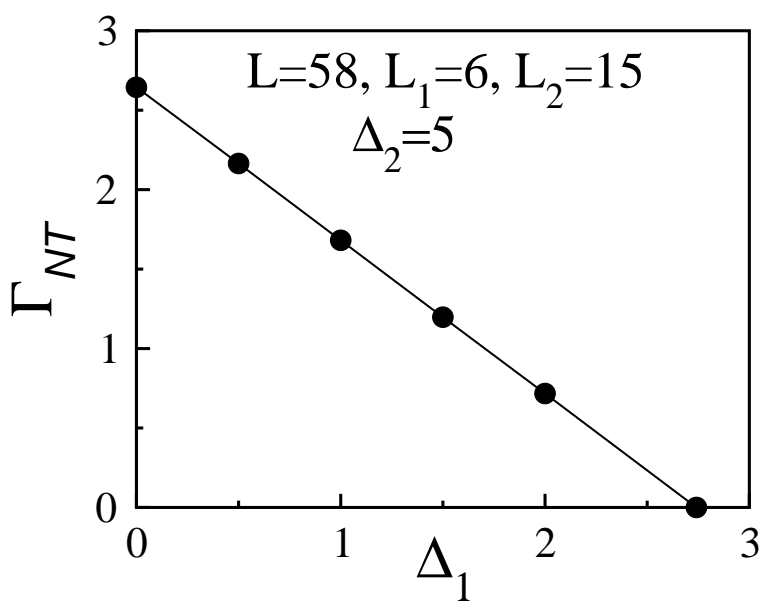

Figure 4: The width of nontrivial plateau $\Gamma_{N T}$ as a function $\Delta_{1}$. In this case $\Delta_{2}=5, L_{1}=6, L_{2}=15(\ell=5 / 2, m=$ $\left.(1+\ell)^{-1}=2 / 7\right), N_{c}=3$, and $L=58$.

In Fig. 3. the magnetization profile at the plateau of $m=0.4$ is shown. The parameters used are $L=100$ $\left(L_{1}=2 P, L_{2}=3 P, P=7\right.$, and $\left.N_{c}=3\right), \ell=3 / 2$, $\Delta_{1}=0.5$, and $\Delta_{2}=5$. We can see that, indeed, in the sub-lattices with anisotropy parameter $\Delta_{1}=0.5$, the spins are fully polarized, whereas the sub-lattices with anisotropy parameter $\Delta_{2}=5$ are antiferromagnetically ordered.

The overall picture resulting from Figs. 2] and 3] and Table凹is thus compatible with the SS having a nontrivial plateau at $m=1 /(1+\ell)$, in which one sub-chain is fully saturated with $m_{1}=1$, whereas the other is in a spingapped AFM phase with $m_{2}=0,{ }^{17.26}$

The width of the plateau of a $\mathrm{SS}$ with $\Delta_{2}=5$ is shown in Fig. 4 as a function of the anisotropy parameter $\Delta_{1}$. The other parameters are $L_{1}=2 P, L_{2}=5 P(\ell=5 / 2$, $\left.m=(1+\ell)^{-1}=2 / 7\right), P=3, N_{c}=3$, and $L=58$. We 


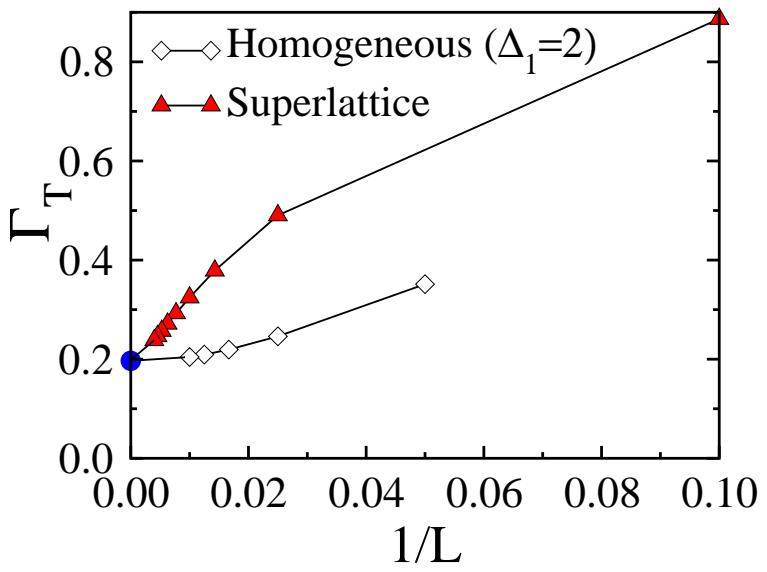

Figure 5: The width of the plateau at zero magnetization as a function of the lattice size for a SS with $\Delta_{1}=2, \Delta_{2}=5$ and $\ell=3 / 2\left(L_{1}=2 P, L_{2}=3 P\right.$, and $\left.N_{c}=3\right)$ (closed triangles). For comparison, we also show the size dependence of the spin gap of a homogeneous chain with anisotropy parameter $\Delta=2$ (open diamonds). The extrapolated infinite size limits of the two curves (closed circles) coincide within the numerical error.

observe that the width of the plateau decreases linearly with $\Delta_{1}$ and vanishes at $\Delta_{1}=2.741$. The magnetization curve of this SS does not have plateaus for $\Delta_{1}>2.741$. This linear dependence is expected. As we have seen, the plateau width is given by $\Gamma_{N T}=h_{c}^{P}\left(\Delta_{2}=5\right)-1-\Delta_{1}$, where $h_{c}^{P}$ is the gap of the corresponding homogeneous spin chain in the AFM phase ${ }^{17}$ The slope of the line in the Fig. 4 is -0.966 , close to the expected value of -1 .

The magnetization curve in Fig. 2 has one nontrivial plateau at $m=0.4$ for the parameters $\Delta_{1}=0.5$ and $\Delta_{2}=5$. When both $\Delta_{1}>1$ and $\Delta_{2}>1$, however, a new plateau at $m=0$ emerges. In this case, both sub-chains 1 and 2 are in a spin-gapped AFM phase 17 For a SS with parameters $\Delta_{1}=2, \Delta_{2}=5$, and $\ell=$ $3 / 2\left(L_{1}=2 P, L_{2}=3 P\right.$, and $\left.N_{c}=3\right)$, the width of the plateau at $m=0$ is shown in Fig. 5 as a function of $1 / L$. Using the VBS algorithm, we determined the infinite size limit of the plateau width to be $\Gamma_{T}=0.198$. According to the analysis of Ref. 17, this value should be given by the gap of a homogeneous spin chain with anisotropy parameter $\Delta_{1}=2$. For comparison, we also show in Fig. [5 the value of the latter gap as a function of system size. The extrapolated gap size is $\Gamma_{T}=0.196$, while the value obtained from the exact solution is $\Gamma_{T}=$ 0.19842. Indeed, the extrapolated values coincide within the numerical error.

\section{GAPLESS REGION: THE EFFECTIVE TOMONAGA-LUTTINGER PARAMETERS}

The low-energy properties of the SS away from the plateaus can be described in terms of a TLLS. ${ }^{19}$ The
Hamiltonian of TLLS is given by

$H_{L L S L}=\frac{1}{2 \pi} \int d x\left\{u(x) K(x)\left(\partial_{x} \Theta\right)^{2}+\frac{u(x)}{K(x)}\left(\partial_{x} \Phi\right)^{2}\right\}$

where $\partial_{x} \Theta$ is the momentum field conjugate to $\Phi$ : $\left[\Phi(x), \partial_{y} \Theta(y)\right]=i \delta(x-y)$. The fields $\Phi$ and $\Theta$ can be related to the spin density operators ${ }^{17}$ In the Hamiltonian (4), we have introduced the sub-chain-dependent parameters $u(x)$ and $K(x)$. For $x$ in the sub-chain $\lambda$, one has $K(x)=K\left(J, \Delta_{\lambda}, h\right)$ and $u(x)=u\left(J, \Delta_{\lambda}, h\right)$, i.e., the usual uniform Tomonaga-Luttinger parameters for each sub-chain, which can be obtained directly from the Bethe Ansatz solution. ${ }^{10.23}$ Of course, this effective low-energy description is valid asymptotically in the limit of very long sub-chains. Using periodic boundary conditions and diagonalizing the Hamiltonian (4), we find that the low energy properties of the SS are determined by just a few effective parameters ${ }^{17.19}$ These parameters are the effective velocity $c$ and the effective correlation exponents $K^{*}$ and $\bar{K}$, which are given by 17,19

$$
\begin{gathered}
c=\frac{u_{1}(1+\ell)}{\sqrt{1+\eta \ell u_{1} / u_{2}+\left(\ell u_{1} / u_{2}\right)^{2}}}, \\
K^{*}=\frac{\sqrt{1+\eta \ell u_{1} / u_{2}+\left(\ell u_{1} / u_{2}\right)^{2}}}{\frac{1}{K_{1}}+\ell \frac{1}{K_{2}} \frac{u_{1}}{u_{2}}} \equiv f\left(K_{1}, K_{2}\right), \\
\bar{K}=f\left(1 / K_{1}, 1 / K_{2}\right),
\end{gathered}
$$

where $\eta=K_{1} / K_{2}+K_{2} / K_{1}$. Clearly, $\left(c, K^{*}, \bar{K}\right) \rightarrow$ $\left(u_{2}, K_{2}, 1 / K_{2}\right)$ as $\ell \rightarrow \infty$, and $\left(c, K^{*}, \bar{K}\right) \rightarrow$ $\left(u_{1}, K_{1}, 1 / K_{1}\right)$ as $\ell \rightarrow 0$, as expected. The important feature to notice in Eqs. (5), (6), and (7) is the fact that the effective SS parameters represent a certain weighted average of the individual sub-chain velocities and correlation exponents. This weighted average is induced by the superlattice structure and is a feature ubiquitous in TLLS's, 17.19

It is straightforward to extract from the Hamiltonian (4) the finite-size spin gap of the system. It is given by

$$
E\left(S_{\text {tot }}^{z}=1, h=0\right)-E\left(S_{\text {tot }}^{z}=0, h=0\right)=\frac{\pi c}{2 K^{*} L} .
$$

Thus, from the scaling of the spin gap with the system size, we can verify the predictions of Eqs. (5) and (6) for the effective Tomonaga-Luttinger parameters.

Fig. [6] shows the numerically determined ratio $c / K^{*}$ for a SS with $\Delta_{1}=0.4$ and $\Delta_{2}=0.8$ as a function of $\ell$. We used $N_{c}=4$ and $L=100,76,100,52,76,100$, for $\ell=1 / 2,3 / 4,5 / 4,3 / 2,5 / 2,7 / 2$, respectively. For comparison, we also show the TLLS prediction obtained from the ratio of Eqs. (5) and (6) and from the known values of $u_{\lambda}$ and $K_{\lambda}$ for homogeneous chains. We can see 


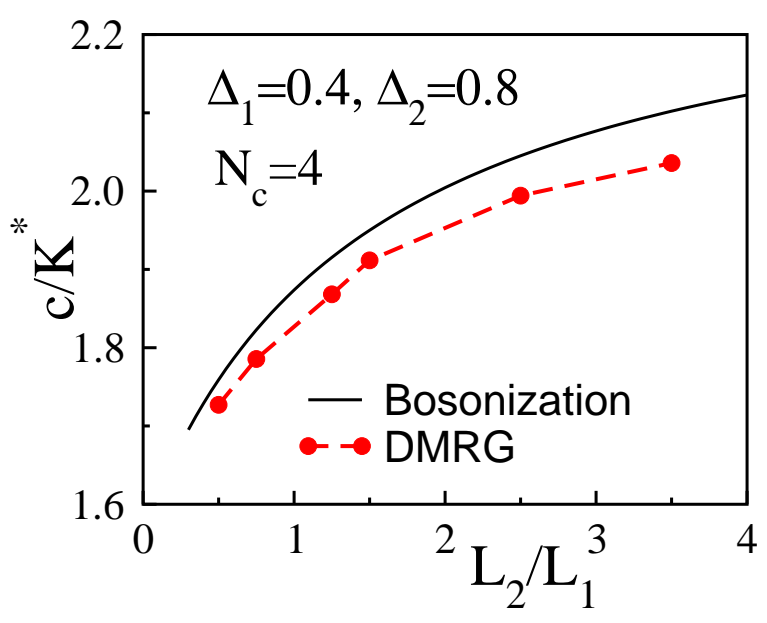

Figure 6: $\quad c / K^{*}$ as a function $\ell$ for a SS with $\Delta_{1}=0.4$ and $\Delta_{2}=0.8$. The continuous line was obtained using bosonization ${ }^{17}$ The closed dots correspond to the DMRG results.

that there is reasonable agreement, with slightly larger discrepancies at larger $\ell$. The ratio $u / K$ for homogeneous chains with anisotropy parameters $\Delta_{1}=0.4$ and $\Delta_{2}=0.8$ are equal to $u_{1} / K_{1}=1.57$ and $u_{2} / K_{2}=2.33$, respectively. $c / K^{*}$ interpolates smoothly between $u_{1} / K_{1}$ and $u_{2} / K_{2}$ as $\ell$ increases, a manifestation of the spatial averaging due to the superlattice structure. We believe the small discrepancies between the curves in Fig. 6] are due to the finite sizes of the sub-chains. We recall that the TLLS predictions are expected to hold asymptotically for very long sub-chains. For a gapless phase, the inhomogeneities created by the boundaries between subchains will give rise to Friedel oscillations which die out only as power laws 27 These disturbances are expected to give rise to finite-size corrections to the TLLS predictions. We stress, however, that although the TLLS analysis predicts a sort of weighted average for the de- pendence of $c / K^{*}$ on $\ell$, the detailed form of this average is highly nontrivial. Yet, precisely this non-linear dependence is strikingly confirmed by the numerical data. We consider this as a stringent test of the predictions of the theory.

\section{CONCLUSIONS}

In summary, we have used the finite size DMRG method with open and periodic boundary conditions to study spin superlattices made up of a periodic arrangement of two XXZ chains with different parameters and sizes. We confirmed previous analytical predictions of a nontrivial plateau in the magnetization curve at $m=1 /(1+\ell)$, where $\ell$ is the relative size of the sub-chains. When both anisotropies are larger than 1 , we have also confirmed the expected trivial plateau at $m=0$. The nontrivial plateau width was shown to approach the asymptotic value of a superlattice with long sub-chains. Moreover, the magnetization profile was seen to be in accord with the analytical predictions of one sub-chain being saturated and the other one being AFM ordered. Finally, we found fairly good agreement in the gapless region with the results of a Tomonaga-Luttinger liquid theory as applied to a superlattice structure. This agreement confirms the non-trivial prediction of this theory for the way the individual sub-chain properties are averaged over in the effective low-energy description of the superlattice.

\section{Acknowledgments}

J. Silva-Valencia is grateful to A. L. Malvezzi for some help with the DMRG method. This work was supported by FAPESP through grants 01/07778-0 (J. S.V.), 00/02802-7 (J. C. X.) and 01/00719-8 (E. M.), and by CNPq through grant 301222/97-5 (E. M.).
1 E. Dagotto, S. Yunoki, A. L. Malvezzi, A. Moreo, J. Hu, S. Capponi, D. Poilblanc, and N. Furukawa, Phys. Rev. B 58, 6414 (1998); T. M. Rice, Z. Phys. B 103, 165 (1997); E. Dagotto, Rep. Prog. Phys. 62, 1525 (1999).

${ }^{2}$ F. D. M. Haldane, Phys. Lett. A 93, 464 (1983).

3 F. D. M. Haldane, Phys. Rev. Lett. 50, 1153 (1983).

4 T. Ziman and H. J. Schulz, Phys. Rev. Lett. 59, 140 (1987).

${ }^{5}$ M. Takahashi, Phys. Rev. Lett. 62, 2313 (1989).

6 S. R. White, Phys. Rev. Lett. 69, 2863 (1992).

7 K. Hallberg, X. Q. G. Wang, P. Horsch, and A. Moreo, Phys. Rev. Lett. 76, 4955 (1996).

8 Y. Ajiro, T. Asano, T. Inami, H. Aruga-Katori, and T. Goto, J. Phys. Soc. Jpn. 63, 859 (1994).

${ }^{9}$ W. Shiramura, K. Takatsu, B. Kurniawan, H. Tanaka, H. Uekusa, Y. Ohashi, K. Takizawa, H. Mitamura, and T. Goto, J. Phys. Soc. Jpn. 67, 1548 (1998).
10 D. C. Cabra, A. Honecker, and P. Pujol, Phys. Rev. B 58, 6241 (1998).

11 W. Chen, K. Hida, and H. Nakano, J. Phys. Soc. Jpn. 68, 625 (1999).

12 R. Citro, E. Orignac, N. Andrei, C. Itoi, and S. Qin, J. Phys.: Condens. Matter 12, 3041 (2000).

13 W. Chen, K. Hida, and B. C. Sanctuary, Phys. Rev. B 63, 134427 (2001).

14 M. Oshikawa, M. Yamanaka, and I. Affleck, Phys. Rev. Lett. 78, 1984 (1997).

15 M. Arlego, D. Cabra, and M. Grynberg, Phys. Rev. B 64, 134419 (2001).

16 T. Yamamoto, M. Asano, and C. Ishii, J. Phys. Soc. Jpn. 69, 3965 (2000).

17 J. Silva-Valencia and E. Miranda, Phys. Rev. B 65, 024443 (2002). 
18 C. N. Yang and C. P. Yang, Phys. Rev. 147, 303 (1966); 150, 321 (1966); 150, 327 (1966); 151, 258 (1966).

19 J. Silva-Valencia, E. Miranda, and R. R. dos Santos, J. Phys.: Condens. Matter 13, L619 (2001); J. Silva-Valencia, E. Miranda, and R. R. dos Santos, Phys. Rev. B 65, 115115 (2002).

20 M. S. Gudiksen, L. J. Lauhon, J. Wang, D. C. Smith, and C. M. Lieber, Nature 415, 617 (2002).

21 S. R. White, Phys. Rev. B 48, 10345 (1993).

22 J. M. Luttinger, J. Math. Phys. 4, 1154 (1963); D. C. Mattis and E. H. Lieb, J. Math. Phys. 6, 304 (1965); D. C. Mattis, J. Math. Phys. 15, 609 (1974); A. Luther and I. Peschel, Phys. Rev. B 12, 3908 (1975); J. Sólyom, Adv. Phys. 28, 201 (1979); V. J. Emery, in Highly Conducting One-Dimensional Solids, edited by J. T. Devreese, R. P. Evrard, and V. E. van Doren (Plenum, New York, 1979), chap. 6, pp. 247-303; R. Heidenreich, R. Seiler, and A. Uhlenbrock, J. Stat. Phys. 22, 27 (1980); F. D. M. Haldane, J. Phys. C 14, 2585 (1981).

23 F. D. M. Haldane, Phys. Rev. Lett. 45, 1358 (1980).

24 D. C. Cabra, M. D. Grynberg, A. Honecker, and P. Pujol, cond-mat/0010376 (unpublished).

25 J. M. Vanden Broeck and L. W. Schwartz, SIAM (Soc. Ind. Appl. Math.) J. Math. Anal. 10, 658 (1979); M. N. Barber and C. J. Hamer, J. Aust. Math. Soc. B 23, 229 (1982).

26 Because of the small sizes, we have kept the AFM subchains with an odd number of sites, such that no frustration of the AFM order is introduced. This is expected to be irrelevant for long sub-chains.

27 R. Egger and H. Grabert, Phys. Rev. Lett. 75, 3505 (1995). 